\title{
铜催化不对称烯丙基烷基化反应的研究进展
}

\author{
崔朋雷* 刘海燕张冬暖 王 春 \\ (河北农业大学理学院 保定 071001)
}

\begin{abstract}
摘要 铜催化不对称烯丙基烷基化反应是一种重要的合成手性化合物的方法，综述了铜催化不对称烯丙基烷基化反应 的最新研究进展, 重点讨论了烯丙基衍生物结构、手性配体结构及亲核试剂类型等因素对该类型反应的影响.

关键词 铜催化; 手性化合物; 烯丙基衍生物; 不对称烯丙基烷基化反应
\end{abstract}

\section{Progress in Copper-Catalyzed Asymmetric Allylic Alkylation Reaction}

\author{
Cui, Penglei* Liu, Haiyan Zhang, Dongnuan Wang, Chun \\ (College of Science, Agricultural University of Hebei, Baoding 071001)
}

\begin{abstract}
Copper-catalyzed asymmetric allylic alkylation reaction is one of the most important methods for preparing chiral compounds. The recent progress of copper-catalyzed asymmetric allylic alkylation reaction is reviewed. Great attention is paid to the influences caused by the structure of allylic derivatives and chiral ligands and the type of nucleophiles on this kind of allylic alkylation reaction.
\end{abstract}

Keywords copper-catalysis; chiral compound; allylic derivative; asymmetric allylic alkylation reaction

不对称烯丙基烷基化反应是一种很重要的增长碳 链的反应, 通过该反应可以合成一些复杂的有机分 子 $^{[1,2]}$ 以及药物的中间体 ${ }^{[3]}$. 人们在该反应中应用了各种 催化剂(如 $\mathrm{Pb}, \mathrm{W}, \mathrm{Mo}, \mathrm{Ir}, \mathrm{Ni}, \mathrm{Rh}, \mathrm{Ru})^{[4 \sim 6]}$, 其中应用最广 泛的是钯催化剂. 但是研究发现钯催化该反应所得到的 产物立体选择性较差, 这就限制了钯催化剂在不对称烯 丙基烷基化反应中的应用. 铜作为一种廉价而且低毒的 金属可以催化多种有机化学反应 ${ }^{[79]}$, 近些年来的研究 发现铜能够催化一些稳定性差的亲核试剂(如有机锂、 有机镁、有机锌试剂)参与的不对称烯丙基烷基化反 应 $^{[10,11]}$. 本文综述了近年来铜催化的不对称烯丙基烷基 化反应的最新研究进展.

烯丙基烷基化反应过程包括两种不同的反应机理: 一种是 $\alpha$-取代, 进攻试剂直接进攻离去基团的 $\alpha$-碳原 子, 该机理属于 $\mathrm{S}_{\mathrm{N}} 2$ 反应; 第二种是 $\gamma$-取代, 可以看作 $\mathrm{S}_{\mathrm{N}} 2^{\prime}$ 反应, 反应中在离去基团断键离开时伴随着碳碳双 键重排(Scheme 1). 两种不同的反应机理得到两种不同 结构的产物, 不同反应条件也会对产物的立体结构产生 影响, 比如反应物的结构、离去基团结构、溶剂、反应 温度、有机金属化合物结构等.

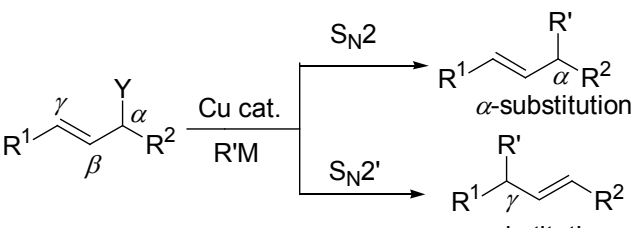

$$
\begin{aligned}
& \mathrm{R}^{\prime}=\text { alkyl, aryl, vinyl, allyl } \\
& \mathrm{M}=\mathrm{Li}, \mathrm{MgX}, \mathrm{Ti}(\mathrm{OR})_{3}, \mathrm{ZnX} \text {, etc. } \\
& \mathrm{Y}=\mathrm{Cl}, \mathrm{Br}, \mathrm{OC}(\mathrm{O}) \mathrm{R}, \mathrm{SO}_{2} \mathrm{Ph}, \mathrm{OR}, \mathrm{OP}(\mathrm{O})(\mathrm{OR})_{2}
\end{aligned}
$$

\section{Scheme 1}

人们对铜催化的烯丙基烷基化反应机理做了深入 研究, 发现该反应按 $\mathrm{S}_{\mathrm{N}} 2$ '机理反应时主要得到构型翻转 的产物 ${ }^{[12 \sim 14]}$ (Eq. 1). 1984年, Corey等 ${ }^{[15]}$ 对于该现象在理 论上用前线分子轨道理论做了合理解释.

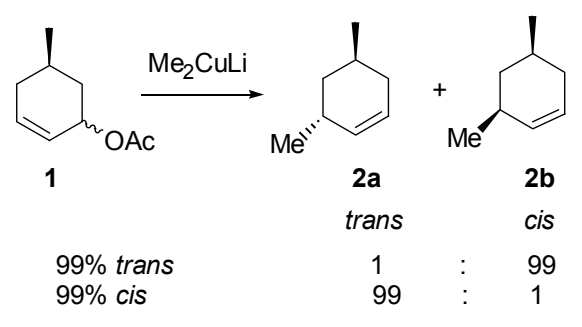

* E-mail: 936369081@qq.com

Received October 7, 2011; revised January 4, 2012; published online March 8, 2012. 
近些年，人们提出了许多关于铜催化的烯丙基烷基 化反应的反应机理 ${ }^{[16 ~ 18]}$, 其中被人们普遍接受的是由 Bäckvall 和 van Koten 等 ${ }^{[19]}$ 提出的 $\mathrm{Cu}(\mathrm{III})$ 过渡态理 论(Scheme 2). 尽管，该反应机理中的 $\mathrm{Cu}(\mathrm{III})$ 过渡态目 前还没有被分离得到或通过光谱学方法检测到, 但是 Bäckvall 等 ${ }^{[20]}$ 却通过其他间接的方法提供了其存在的证 据.

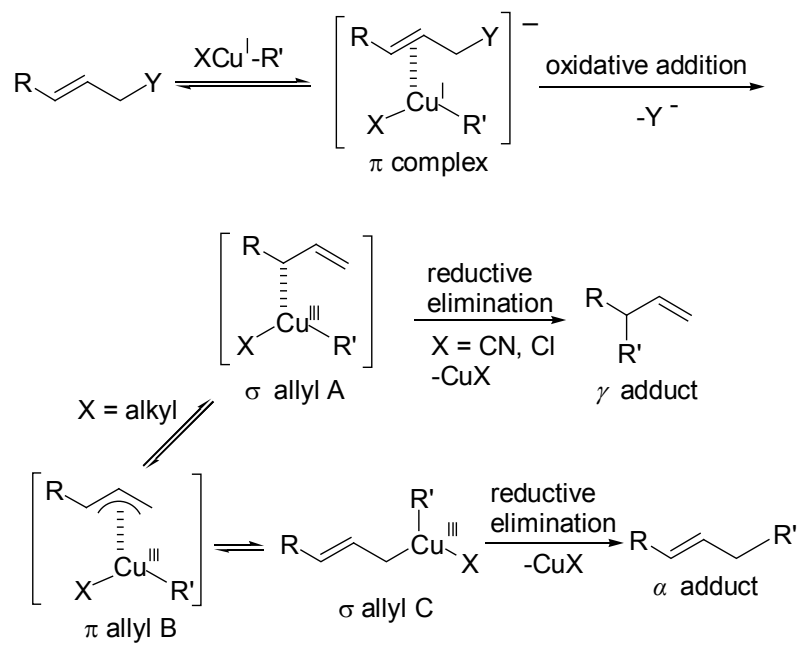

\section{Scheme 2}

\section{1 有机镁试剂}

1995 年 Bäckvall 和 van Koten 等 ${ }^{[21]}$ 首次报道了铜催 化的不对称烯丙基烷基化反应. 经过分析离去基团结 构、 $\mathrm{R}$ 取代基结构、反应温度、有机金属试剂加入的方 法，他们总结了控制不对称 $\mathrm{S}_{\mathrm{N}} 2^{\prime}$ 反应产物立体结构的一 些困难, 提出了一些解决方法. 烯丙基酯 3 和碘化丁基 镁 4 进行 $\gamma$ 烷基化反应中增加 CuSAr 的用量可提高反应 产物的立体专一性, 产物的 $e e$ 值为 $28 \% \sim 42 \%$. 他们注 意到该反应中缓慢地同时添加格氏试剂和底物条件下 反应产物选择性会更高, 并且该反应在低于 $0{ }^{\circ} \mathrm{C}$ 条件 下反应主要得到构型相反的产物(Eq. 2).

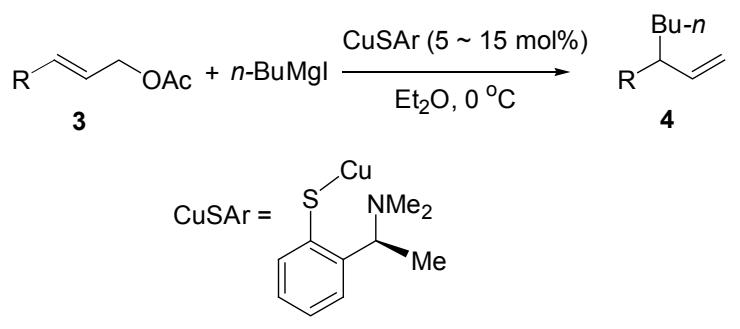

complete $\gamma$-selectivity

$\mathrm{R}=c-\mathrm{Hex}(5), 42 \%$ ee (+)

$\mathrm{R}=\mathrm{CH}_{2} \mathrm{OPh}(6), 34 \%$ ee

$\mathrm{R}=\mathrm{Ph}(7), 28 \%$ ee
随后含溴的格氏试剂被用于上述反应，产物的 $e e$ 值较高, 如溴化正丁基镁与环已基取代的烯丙基乙酸酯 8 反应，产物的 $e e$ 值可达 $50 \%$. 反应中如果用空间位阻 更大的有机镁试剂 $\left(\mathrm{Me}_{3} \mathrm{SiCH}_{2} \mathrm{MgI}\right)$ 可使 $\gamma$ 取代产物 9 的 $e e$ 值达到 $53 \%$ ，但产物产率较低 ${ }^{[22]}$ (Eq. 3).
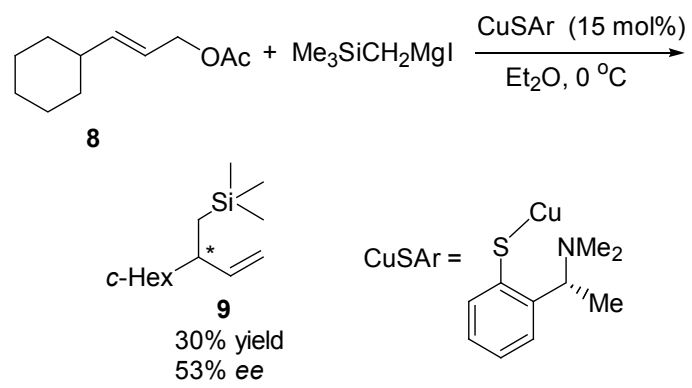

Bäckvall 等 ${ }^{[23]}$ 将含二茂铁的手性配体 $\mathbf{L 1}$ 和 $\mathrm{CuI}$ 作 催化剂用于催化烯丙基烷基化反应，结果发现在该条件 下环己基取代的烯丙基乙酸酯(10)和正丁基碘化镁在乙 醚和甲苯 $[V($ 乙醚 $): V($ 甲苯 $)=3: 1$ ] 混合溶剂中在室温 条件下反应, 所得产物产率较高, 并且产物 ee 值可达 64\% (Eq. 4).

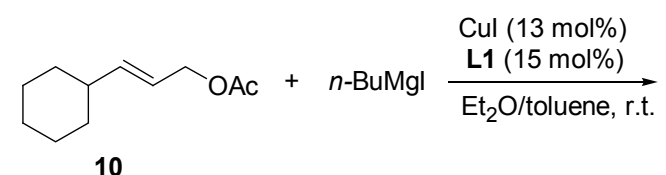

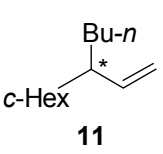

$88 \%$ yield $64 \%$ ee

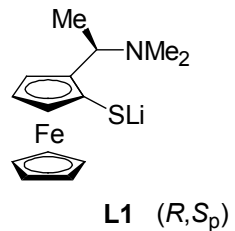

L1 $\left(R, S_{p}\right)$
Alexakis 等 ${ }^{[24]}$ 首次将手性芳基磷配体 $\mathbf{L 2}$ 用于有机 镁试剂参与的烯丙基取代反应. 他们在实验中考查了多 种不同结构的含磷配体，结果发现苯基烯丙基氯(12)与 乙基溴化镁在含磷配体 $\mathbf{L 2}$ 与 $\mathrm{CuCN}$ 作用下反应，得到 产物 13 的 $e e$ 值可达 $73 \%$. 他们还发现反应时减缓有机 镁试剂添加速度有助于提高反应产物的对映选择性(Eq. 5). 随后 Alexakis 等 ${ }^{[25]}$ 用铜噻吩羒酸 $(\mathrm{CuTC})$ 替代反应中 的 $\mathrm{CuCN}$ 与 $\mathbf{L 2}$ 存在下进行该反应, 发现产物 $\mathbf{1 3}$ 的 $e e$ 值由 $73 \%$ 升高到 $82 \%$. 随后他们将含磷配体 $(R, R)-\mathbf{L} 3$ 用 于 $i-\mathrm{PrMgBr}$ 与底物 14 的反应, 得到满意的结果, $\gamma$ 取代 产物 15 达到 91\%, 产物的 $e e$ 值达到 86\% (Eq. 6). 铜催 化的不对称烯丙基烷基化反应中底物应用较多的是芳 香族烯丙基氯(如 12,14), 后来脂肪族烯丙基氯 16 也被 应用到该反应中, 如底物 $\mathbf{1 6}$ 与 $i-\mathrm{PrMgBr}$ 在 $\mathrm{CuTC}$ 与含 磷配体 $(R, S, S)-\mathbf{L} \mathbf{4}$ 作用下反应，所得到产物 $\mathbf{1 7}$ 的 $e e$ 值可 达 74\% (Eq. 7). 


$$
\overbrace{12} \mathrm{Cl}+\mathrm{EtMgBr} \frac{\stackrel{\mathrm{CuX}(1 \mathrm{~mol} \%)}{\mathrm{L2}(1 \mathrm{~mol} \%)}}{\mathrm{CH}_{2} \mathrm{Cl}_{2},-80{ }^{\circ} \mathrm{C}} \overbrace{13} \overbrace{13}^{\mathrm{Et}}
$$<smiles>CN(C)C([N])C(OP1OC(c2ccccc2)(c2ccccc2)C(c2ccccc2)C(c2ccccc2)(c2ccccc2)O1)N(C)C</smiles>

\section{L2}

$\mathrm{CuX}=\mathrm{CuCN}, \gamma / \alpha=94: 6,73 \%$ ee $(R)$

CuTC, $\gamma / \alpha=96: 4,82 \%$ ee $(R)$

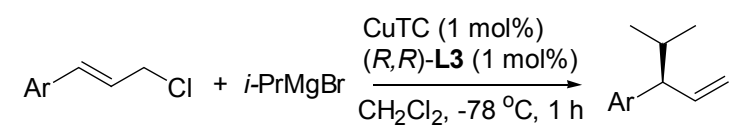

14

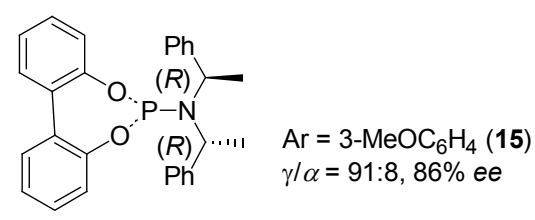

L3<smiles>ClC/C=C/C1CCCCC1</smiles>

16

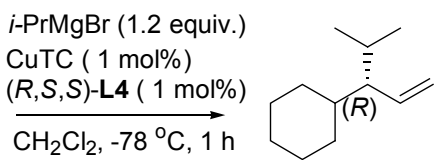

17

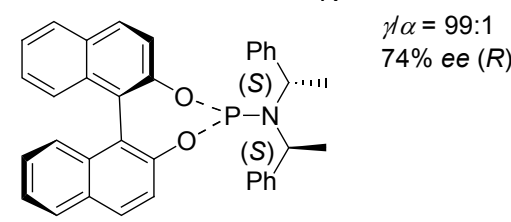

L4

最近，一种新的氨基亚磷酸盐手性配体 $(R, R, R)-\mathbf{L 5}$ 被用于铜催化的烯丙基烷基化反应中, 其具有很好的立 体和对映选择性 ${ }^{[26,27]}$. 配体 $(R, R, R)-\mathbf{L 5}$ 为具有半活性的 $\mathrm{P}, \mathrm{O}$ 双齿配体, 它与 $\mathrm{CuTC}$ 催化的底物 $\mathbf{1 4}$ 与有机镁试剂 的反应, 所得产物具有很高的对映选择性, 产物 $e e$ 值最 高可达 $96 \%$, 该反应产物可在手性碳原子构型保持情况 下进一步反应形成环状化合物(Scheme 3). 此外, 配体 $(R, R, R)-\mathbf{L 5}$ 还适用于催化有机锌(如二乙基锌)参与的烯 丙基烷基化反应.

随后, 具有高对映选择性的催化体系 CuTC/L5 被 成功用于催化不同结构的烯丙基氯甲基化反应, 产物 $e e$ 值一般大于 $90 \%{ }^{[28]}$, 这种方法被用于合成一种具有消炎 功能手性药物耐普生(Naproxen)的前体 22 (Eq. 8).

后来, Alexakis 等 ${ }^{[29]}$ 将氨基亚磷酸盐手性配体 $(S, S, S)$ - L6 用于烯丙基烷基化反应，发现底物不管是芳 香结构还是脂肪族结构取代的烯丙基氯, 所得到产物具 有很好的立体和对映选择性, 并且不管底物是富电子芳

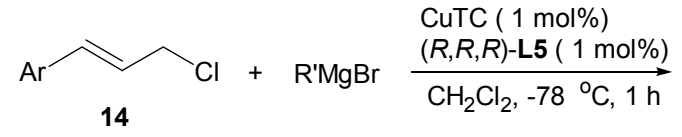<smiles>[R]C([Al])C=C</smiles>

up to $96 \%$ ee $(R)$ for $\mathrm{R}^{\prime}=\mathrm{Et}(\mathbf{1 8})$ $\mathrm{R}^{\prime}=4$-pentenyl $(19 \mathrm{a})$ up to $93 \%$ ee $(R)$ for $R^{\prime}=$ 3-butenyl $(19 b)$

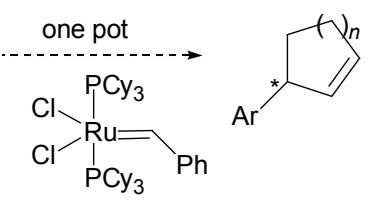

up to $96 \%$ ee $(R)$ for $n=2(20 \mathrm{a})$ up to $93 \%$ ee $(R)$ for $n=1$ (20b)

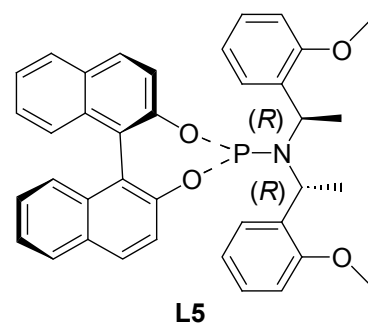

\section{Scheme 3}

$$
\begin{aligned}
& \mathrm{MeMgBr} \text { ( } 3 \text { equiv.) } \\
& \mathrm{CuBr} \text { (5 mol\%) } \\
& \mathrm{Cl} \frac{(R, R, R)-\mathbf{L} 5(5.5 \mathrm{~mol} \%)}{\mathrm{CH}_{2} \mathrm{Cl}_{2},-78{ }^{\circ} \mathrm{C}}
\end{aligned}
$$

o

21<smiles>C=CC(C)(C)c1ccc2cc(OC)ccc2c1</smiles>

$90: 10$ $93 \%$ ee

香结构还是缺电子芳香结构, 所得到的产物 $e e$ 值都较 高,最高可达 98\% (Eq. 9). 除此之外含有脂肪环烯烃结 构的烯丙基氯作为底物进行反应，所得的产物的 $e e$ 值 也很高(Eq. 11).

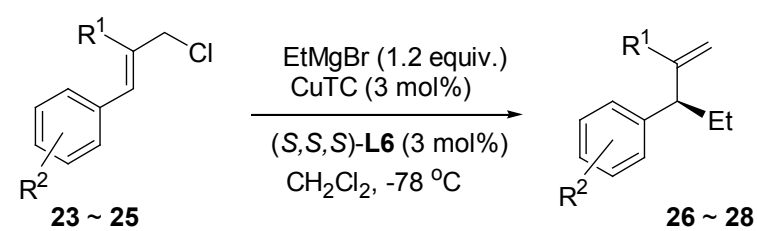<smiles>CC(c1ccccc1)N(C)P(Oc1ccc2ccccc2c1-c1ccccc1)Oc1ccc2ccccc2c1-c1ccccc1</smiles>
$\mathrm{R}^{1}=\mathrm{Me}, \mathrm{R}^{2}=\mathrm{H}(\mathbf{2 6}) 86 \%$ yield yl $\alpha=92: 8,98 \%$ ee $(+)$ $\mathrm{R}^{1}=\mathrm{Me}, \mathrm{R}^{2}=\mathrm{Cl}(\mathbf{2 7}) 87 \%$ yield भl $\alpha=92: 8,96 \%$ ee (+) $\mathrm{R}^{1}=\mathrm{Et}, \mathrm{R}^{2}=\mathrm{H}(\mathbf{2 8}) 87 \%$ yield $\gamma \alpha=83: 17,92 \%$ ee (+) 

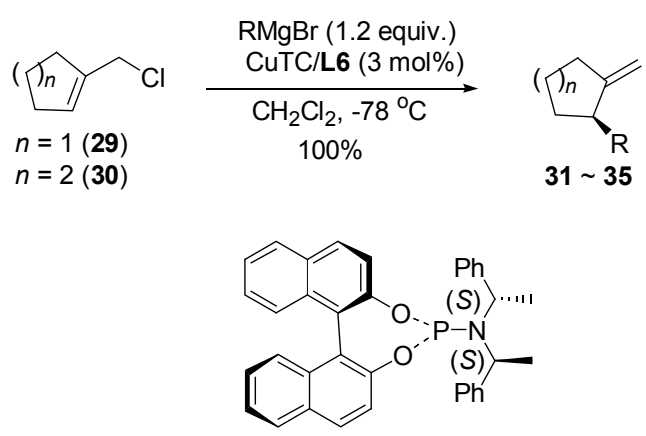

L6
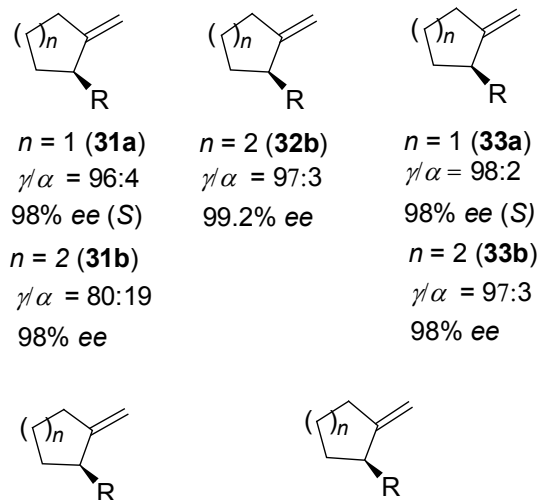

$n=1(34 a)$

$\gamma / \alpha=97: 3$

$98 \%$ ee

$n=2(34 b)$

$\gamma / \alpha=85: 15$

$99.4 \%$ ee

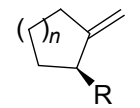

$n=1(35 \mathrm{a})$

$\gamma / \alpha=98: 2$

$98 \%$ ee

$n=2(35 b)$

$\gamma / \alpha=91: 9$

$98.8 \%$ ee
Okamoto 等 ${ }^{[30,31]}$ 在不对称烯丙基烷基化反应中未使 用含磷配体而是使用含 $\mathrm{N}$ 杂环铜配合物 36 作为催化剂. 底物 37 为 $Z$ 构型双官能团化合物, 在催化剂 36 作用下 进行烯丙基烷基化反应可生成产物 38, 产物 ee 值达到 $70 \%$. 若用 $E$ 构型的 37 作为反应物参与反应, 所得产物 构型相反并且产物 $e e$ 值有所降低, 为 $60 \%$ (Eq. 11).
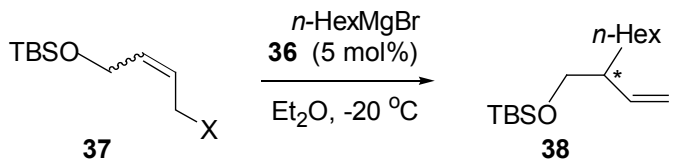

38

for (Z)-substrate $\gamma / \alpha=98: 2,70 \%$ ee $\quad(R)$

for $(E)$-substrate $\gamma / \alpha=86: 14,60 \%$ ee

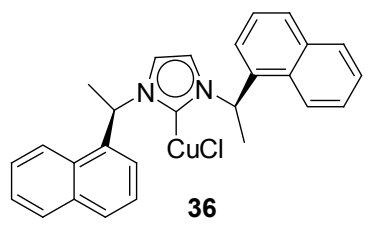

最近, Feringa 研究小组 ${ }^{[32]}$ 发现基于二茂铁的含磷配 体 L7 和铜作催化剂 $-78^{\circ} \mathrm{C}$ 条件下在二氯甲烷溶剂中有机 镁参与的不对称烯丙基烷基化反应, 所得产物具有很高
的立体选择性. 他们还指出在双齿配体 $(R, S)$ - - 7 作用下, 镁试剂与不同结构烯丙基溴进行反应，所得产物中 $\gamma$ 取 代产物所占比例较高并且产物 ee 值最高达 $98 \%$ (Eq. 12). 随后, Feringa 研究小组 ${ }^{[33,34]}$ 又报道了将 $\mathrm{CuBr}$ • $\mathrm{SMe}_{2} / \mathbf{L} 7$ 作为催化剂用于催化多官能团的脂肪族烯丙基 醚 43,45 和氨基甲酸酯 44 及 46 作为底物的烯丙基烷基 化反应. 实验结果发现, 反应具有很高的对映选择性, 产物 $e e$ 值一般都大于 $92 \%$ (Eq. 13).
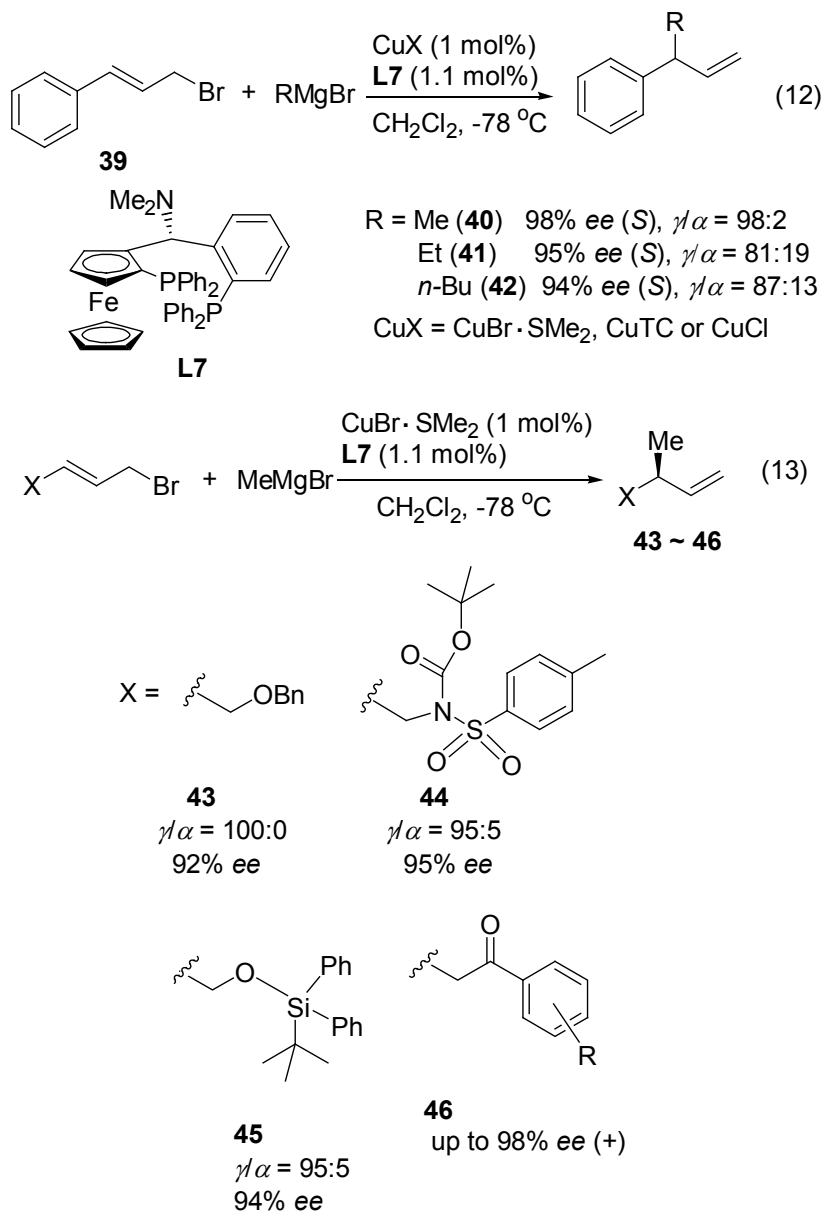

含氮杂环化合物在自然界广泛存在并且在生物体 中发挥着重要的生理作用, 最近 Feringa 等 ${ }^{[35]}$ 报道了一 种利用不对称烯丙基烷基化反应产物合成手性含氮杂 环化合物的方法，在配体 $\mathbf{L} 7$ 与 $\mathrm{CuBr}$ 催化作用下 $\mathrm{N}$ 取代 的烯丙基溴和甲基溴化镁反应主要得到 $\gamma$ 取代产物，该 产物进一步反应得到含氮杂环化合物. 通过该方法成功 和成了六元至八元不饱和的含氮杂环化合物, 值得注意 的是在利用烯丙基烷基化产物进行关环反应所得到的 含氮杂环化合物的 $e e$ 值并未降低(Scheme 4).

最近, Tomioka 等 ${ }^{[36]}$ 将新型含氮杂环配体铜复合物 $\mathbf{5 0}$ 用于催化烷基烯丙基溴 $\mathbf{5 1}$ 与含芳基格式试剂进行的 不对称烯丙基烷基化反应，实验中考查了格氏试剂中芳 基结构、配体中芳基结构、底物 $\mathbf{5 1}$ 及配体空间位阻效 

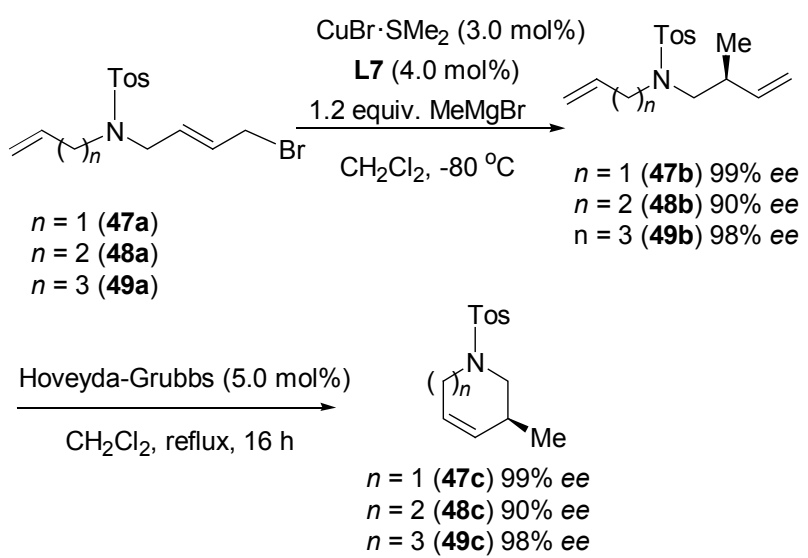

\section{Scheme 4}

应等因素对实验结果的影响后发现该反应以 $\gamma$-取代产 物为主, 可能是由于反应生成的 $\sigma$ 烯丙基铜中间体中因 配体较大的空间位阻及缺电子性而提高了其在下一步 消除反应的速率. 实验结果显示 50a, 50f 用于该反应所 得产物具有较高立体选择性及对映选择性(Eq. 14).

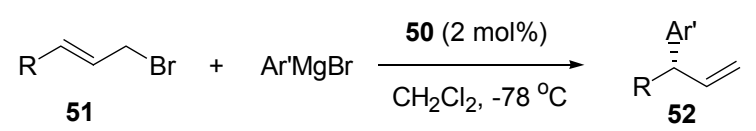<smiles>[Y]C([Al])N1C([Al])N(C([Al])[Al])C(c2ccccc2)[C@H]1c1ccccc1</smiles>

up to $96 \%$ ee up to $96 \% \gamma$-product up to $99 \%$ yield

50a: $\mathrm{Ar}=\mathrm{Ph}, \mathrm{X}=\mathrm{Cl}$; 50f: $\mathrm{Ar}=4-\mathrm{FC}_{6} \mathrm{H}_{4}, \mathrm{X}=\mathrm{Cl}$

50b: $\mathrm{Ar}=2-\mathrm{MeC}_{6} \mathrm{H}_{4}, \mathrm{X}=\mathrm{Cl} ; \mathbf{5 0 g}: \mathrm{Ar}=3-\mathrm{FC}_{6} \mathrm{H}_{4}, \mathrm{X}=\mathrm{Cl}$

50c: $\mathrm{Ar}=2,3-\mathrm{Me}_{2} \mathrm{C}_{6} \mathrm{H}_{3}, \mathrm{X}=\mathrm{TC} ; \mathbf{5 0 h}: \mathrm{Ar}=2-\mathrm{FC}_{6} \mathrm{H}_{4}, \mathrm{X}=\mathrm{Cl}$

50d: $\mathrm{Ar}=2,4-\mathrm{Me}_{2} \mathrm{C}_{6} \mathrm{H}_{3}, \mathrm{X}=\mathrm{TC} ; 50 \mathrm{i}: \mathrm{Ar}=3,5-\mathrm{F}_{2} \mathrm{C}_{6} \mathrm{H}_{3}, \mathrm{X}=\mathrm{Cl}$

50e: $\mathrm{Ar}=2,5-\mathrm{Me}_{2} \mathrm{C}_{6} \mathrm{H}_{3}, \mathrm{X}=\mathrm{TC} ; 50 \mathrm{j}: \mathrm{Ar}=4-\mathrm{CF}_{3} \mathrm{C}_{6} \mathrm{H}_{4}, \mathrm{X}=\mathrm{Cl}$

50k: $\mathrm{Ar}=4-\mathrm{MeC}_{6} \mathrm{H}_{4}, \mathrm{X}=\mathrm{Cl}$

最近, Feringa 等 ${ }^{[37]}$ 将新型含二茂铁的配体 $\mathbf{L 8}$ 用于 不对称烯丙基烷基化反应, 结果发现产物具有很高的立 体选择性与对映选择性. 实验中若将 $(R, R)-\mathbf{L 8}$ 用 $(S, S)$ - - 221 代替反应所得主要产物的构型将发生翻转, 并 且反应温度对产物的立体选择性具有较大影响, 此外底 物结构对产物构型也有至关重要的影响(Eq. 15).

\section{2 有机锌试剂}

继 Bäckvall 与 van Koten 在有机镁试剂参与的烯丙 基烷基化反应中做了开创性工作后, 1999 年 Dubner 与 Knochel 将有机锌试剂成功用于了烯丙基烷基化反 应 $^{[38,39]}$, 实验结果发现含二茂铁手性胺配体 $\mathbf{L 8}$ 与铜 (I) 催化作用下烯丙基氯与有机锌反应所得产物的 $e e$ 值最 高可达 $87 \%$. 该反应产物以 $\gamma$-取代产物为主, 但也发现 具有较大位阻的底物参与反应所得产物产率为 $72 \%$, 该 反应的对映选择性受温度影响很大: 假如反应在室温下

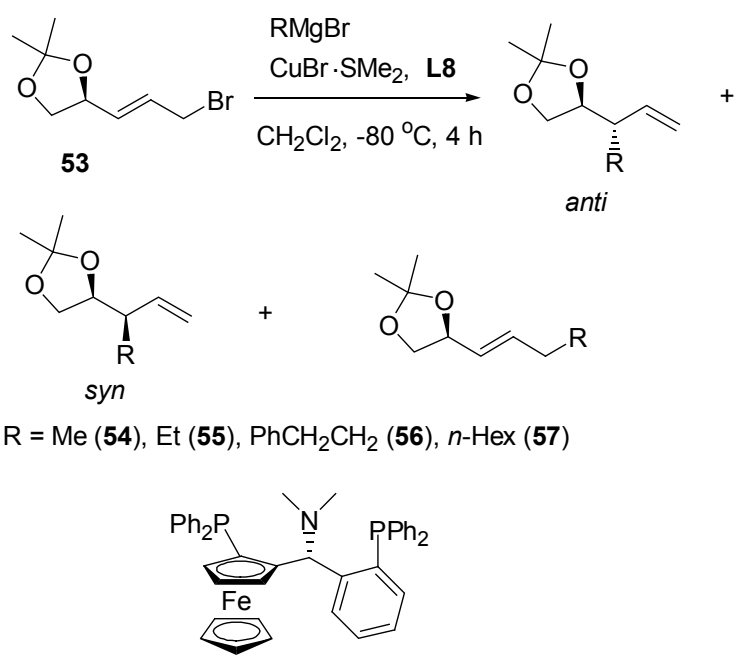

$(R, R)$-L8

进行时产物的 $e e$ 值会由 $87 \%$ 降至 $25 \%$ (Eq. 16). 随后, 在该类反应中应用了结构得到进一步改进的配体 $\mathbf{L 9}$, 并且底物和锌试剂同时加入, 反应 $3 \mathrm{~h}$, 结果发现 $\mathrm{S}_{\mathrm{N}} 2^{\prime}$ 取代产物 $e e$ 值达到了 $98 \%$ (Eq. 17).

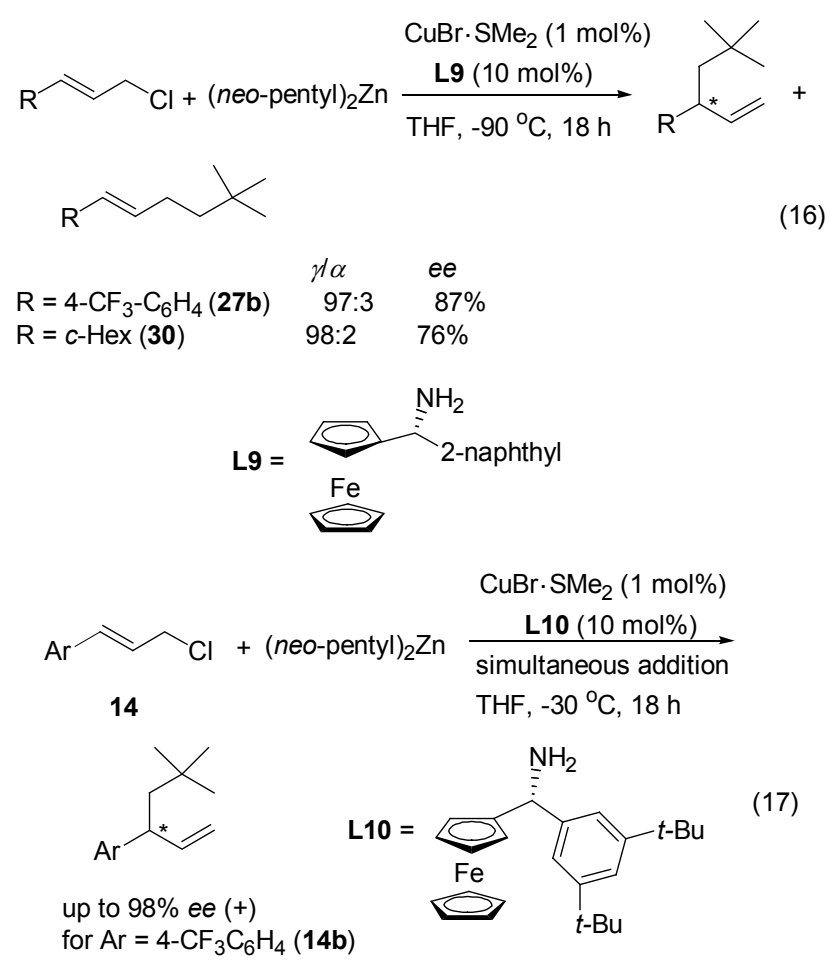

Knochel 将线性的烷基锌试剂用于烯丙基烷基化反 应，所得产物尽管具有较高的对映选择性，但是它的应 用还是面临着很大挑战. Feringa 等 ${ }^{[40]}$ 将低级烷基锌试剂 用于手性含磷配体 L4 作用下不对称烯丙基烷基化反应, 所得产物具有较高的对映选择性(Eq. 18). 通过分析比 较离去基团、反应温度、配体和溶剂对反应的影响, 确 定了在最优条件下烯丙基澳(39)与二乙基锌进行反应, 
所得产物 $e e$ 值为 $77 \%$. 然而, 反应中使用较高极性的溶 剂是否可提高产物的立体选择性还有待进一步的实验 验证. 随后 CuOTf 作为催化剂用于该反应, 溶剂用四氢 呋喃, 含磷配体用 $8 \mathrm{H}-\mathbf{L} 4$, 结果发现二烷基锌与烯丙基 溴 39 反应产物的 $e e$ 值可达 $88 \%{ }^{[41]}$ (Eq. 19).

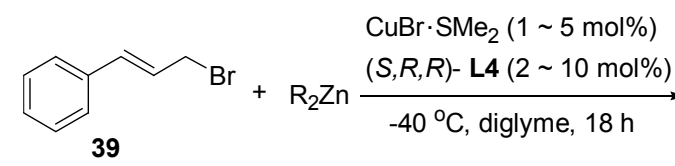<smiles>[R]C(C=C)c1ccccc1</smiles>

$$
\begin{array}{ll}
\mathrm{R}=\mathrm{Et}(\mathbf{4 1}) & \text { up to } 77 \% \text { ee } \\
& \gamma / \alpha=84: 16,70 \% \text { conv. } \\
\mathrm{R}=n-\mathrm{Bu}(\mathbf{4 2}) & 71 \% \text { ee } \\
& \gamma / \alpha=84: 16,65 \% \text { conv. }
\end{array}
$$

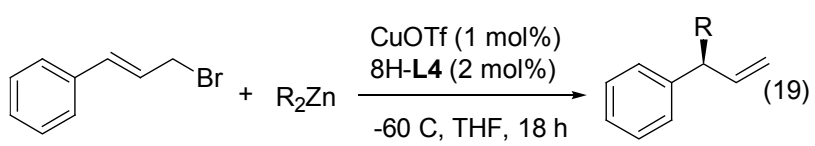

39<smiles>CC(c1ccccc1)P(=P)(Oc1ccc2c(c1-c1ccccc1)CCCC2)N(c1ccccc1)c1ccccc1</smiles>

$\mathrm{R}=\mathrm{Et}(\mathbf{4 1}), \quad \gamma \alpha=93: 7,86 \%$ ee $\mathrm{R}=i-\operatorname{Pr}(\mathbf{5 8}), \quad \not \alpha=97: 3,88 \%$ ee $\mathrm{R}=n-\mathrm{Bu}(\mathbf{4 2}), \quad \gamma \alpha=91: 9,87 \%$ ee

Woodward 等 ${ }^{[42,43]}$ 用 Morita-Baylis-Hillman 反应产 物作为底物与二乙基锌进行不对称烯丙基烷基化反应, 反应中应用 59 作为底物, 二荟酚 L11 作为手性的配体, 结果发现产物具有中等的对映选择性, 如离去基团为 $\mathrm{Cl}$, 芳基为 $4-\mathrm{NO}_{2} \mathrm{C}_{6} \mathrm{H}_{4}$ 时产物 $e e$ 值为 $64 \%$, 并且反应中 需要用较高量的催化剂(10 mol\%)及配体(20 mol\%) (Eq. 20).<smiles>[X]CC(=CBr)C(=O)OCC</smiles>

59<smiles></smiles>

Woodward 等 ${ }^{[44]}$ 在烯丙基烷基化反应中研究了一系 列磷酰胺配体后将手性胺配体 $\mathbf{L 1 2}$ 用于有机锌参与的
不对称烯丙基烷基化反应中. 结果发现二烷基锌参与的 反应中, 产物的 $e e$ 值达到最大值后若延长反应时间会 使得产物 ee 值降低, 这可能是因为反应中逐步生成 $\mathrm{EtZnCl}$ 而降低了反应的选择性. 研究发现反应中使用 甲基铝试剂 $\left\{\mathrm{MAO}:[\mathrm{Al}(\mathrm{Me}) \mathrm{O}]_{n}\right\}$ 可以使产物的 $e e$ 值提高, 还发现底物中含有给电子基团可使产物 $e e$ 值提高, 如 底物连有 4- $\mathrm{MeOC}_{6} \mathrm{H}_{4}$ 产物 $e e$ 值可达 $90 \%$ (Scheme 5).
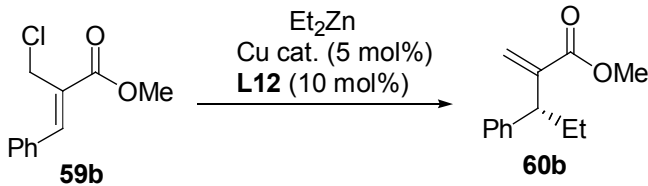

Cul, THF, $-20^{\circ} \mathrm{C}: 78 \%$ ee

CUTC, MAO, DME, $-40^{\circ} \mathrm{C}: 87 \%$ ee
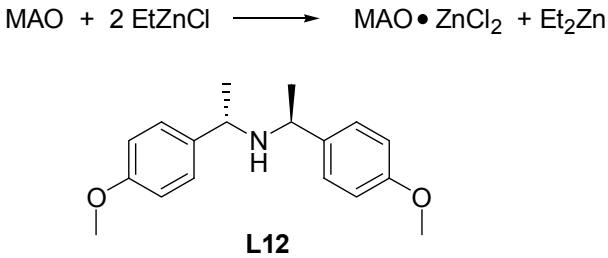

Scheme 5

2001 年, Hoveyda 等 ${ }^{[45]}$ 使用多肽基配体 $\mathbf{L 1 3}$ 与铜催 化剂提高不对称 $\mathrm{S}_{\mathrm{N}} 2^{\prime}$ 反应的选择性, 在 L13 与铜催化剂 作用下二乙基锌与烯丙基磷酸酯反应所得产物的 $e e$ 值 为 $66 \% \sim 90 \%$. 研究还发现, 苯环上连有斥电子基的底 物参与反应所得产物为 $\gamma$ 取代且产物具有较高的对映选 择性(产物 $e e$ 值最高可达 $90 \%$ ). 这是首次报道以磷酸酯 作为底物的不对称烯丙基烷基化反应(Eq. 22). 随后该 方法被成功用于合成某些具有手性的天然产物.

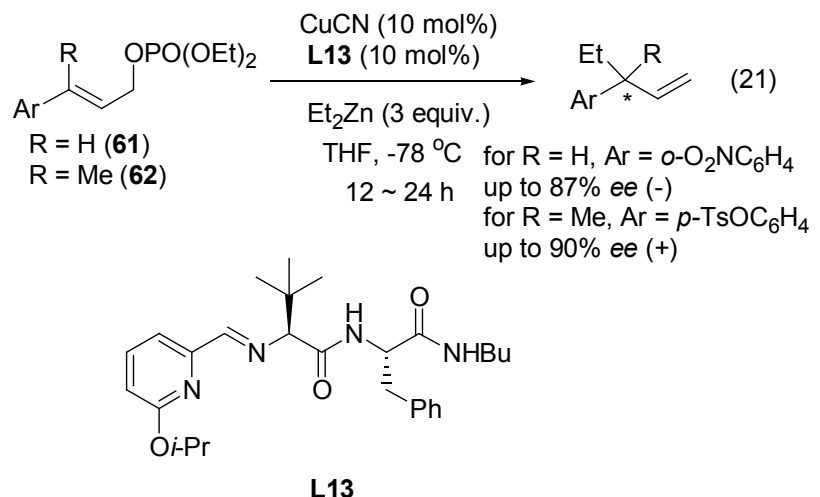

随后，他们对于含多肽的席夫碱在结构上做了修 饰, 得到配体 L14, 并把配体 L14 与铜用于催化不饱和

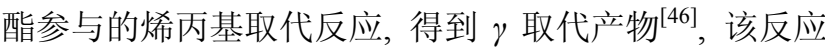
所得产物 $e e$ 值为 $87 \% \sim 97 \%$. 分析实验结果发现该反应 的最佳条件是配体 $\mathbf{L 1 4}$ 与 $(\mathrm{CuOTf})_{2} \cdot \mathrm{C}_{6} \mathrm{H}_{6}$ 一起使用, 随 后研究重点关注不饱和酯作为底物参与反应时对产物 
立体化学结构影响的因素, 结果发现底物中含有叔丁基 或异丙基等具有较高位阻的基团时反应所得主要产物 为 $\gamma$ 取代产物, 并且 $\mathbf{S}_{\mathrm{N}} 2 / \mathbf{S}_{\mathrm{N}} 2$ 产物比例为 $7: 1$ (Eq. 23). 后来, Hoveyda 等 ${ }^{[47,48]}$ 将多肽席夫碱配体 L15 取代 L14 用于上述反应, 结果发现反应产物的对映选择性得到提 高, 产物 $e e$ 值达到 96\%(同样的反应用 L14 作配体产物 $e e$ 值为 $87 \%$ ), 但是同时发现所得产物的立体选择性却 降低了。

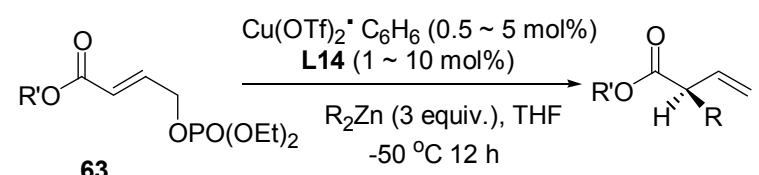

63

up to $92 \%$ ee, $>95 \% \gamma$-selectivity $\mathrm{R}^{\prime}=t-\mathrm{Bu}(63 \mathrm{a})$ or $i-\mathrm{Pr}, \mathrm{R}=\mathrm{Et}(63 \mathrm{~b})$<smiles>CCCCCC(C)C(=O)N[C@@H](CCCC)C(=O)N=Cc1c(O)ccc2ccccc12</smiles>

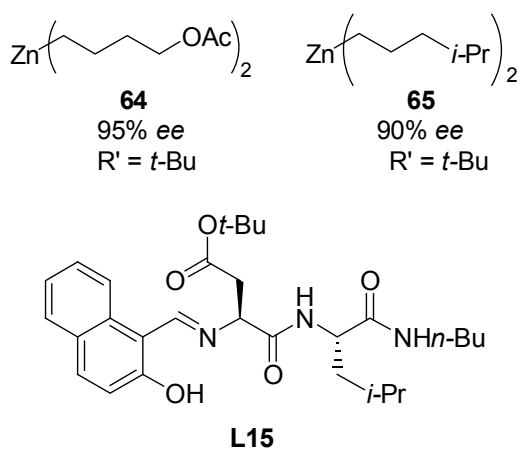

Gennari 等 ${ }^{[49}$ 报道了手性配体 L16, L17 用于内消旋 底物 62 与二烷基锌的反应, 如在铜(I)复合物和配体 L16 作用下二乙基锌和环戊烯基二磷酸酯反应, 产物具有良 好的立体选择性和非对映选择性; 反应产物全部是 $\mathrm{S}_{\mathrm{N}} 2^{\prime}$ 取代产物, 二甲基锌参与的反应所得产物的 $e e$ 值高达 $94 \%$, 二乙基锌和二苯基锌反应所得产物 $e e$ 值相对较低 分别为 $88 \%$ 与 $68 \%$ (Eq. 23). 后来 Gennari 与 Feringa 合 作将环戊烯基、环己烯基、环庚烯基二磷酸酯作为底物 与二乙基锌在该条件下反应 ${ }^{[50]}$, 结果发现产物的 $e e$ 值 分别为 $87 \%, 94 \%,>98 \%$.

Hoveyda 等 ${ }^{[51}$ 将手性配体 L18 用于铜催化的烯丙基 烷基化反应，这种催化体系比之前提到的多肽基配体效 果更好, 反应中配体 $\mathbf{L 1 8}$ 用量较低, 仅需 $2 \mathrm{~mol} \%$ 就可使 二或三取代的烯烃反应高选择性得到产物. 为了增加反 应的效率和选择性又制备了 L18.Ag 复合物, 将它与铜 盐一起使用催化烯丙基烷基化反应, 结果发现产物 ee 值可达到 $98 \%$ (Eq. 24). 后来, Hoveyda 等 ${ }^{[22]}$ 又报道了具
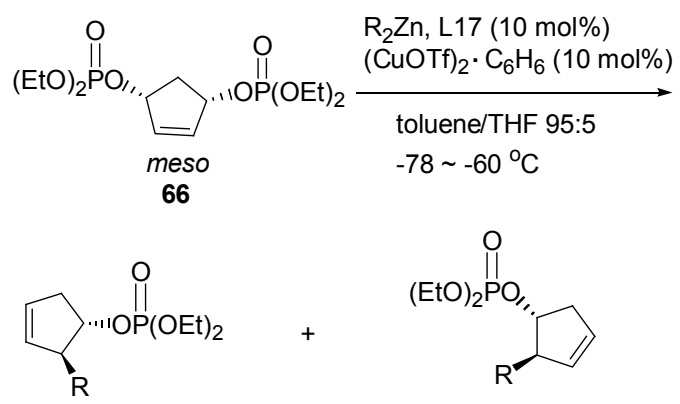

$\mathrm{R}=\mathrm{Me}(67), \gamma / \alpha>99: 1,94 \%$ ee $\mathrm{R}=\mathrm{Et}(\mathbf{6 8}), \gamma / \alpha>99: 1,88 \%$ ee $\mathrm{R}=\mathrm{Ph}(69), \gamma / \alpha>99: 1,68 \%$ ee

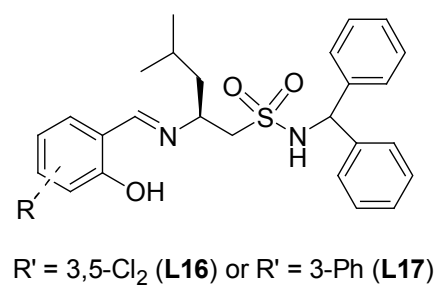<smiles>[R]O[R]OCC=C([R])c1cccc([R])c1</smiles>

$70 \sim 71$<smiles>[R][X]C([R])(C=C)c1ccccc1</smiles>

$$
\begin{array}{ll}
\mathrm{R}=\mathrm{R}^{\prime}=\mathrm{H}(\mathbf{7 0 a}) & 82 \% \text { ee, } 98 \% \gamma \text {-selectivity } \\
\mathrm{R}=\mathrm{Me}, \mathrm{R}^{\prime}=p-\mathrm{NO}_{2} \text { (71a) } & 98 \% \text { ee },>98 \% \gamma \text {-selectivity }
\end{array}
$$<smiles>C[N+]1=CN(c2ccc3ccccc3c2-c2cc(O)cc3ccccc23)CC1</smiles>

有双官能团的乙烯基硅烷 72, 73 参与的烯丙基烷基化反 应，结果发现所得产物 $e e$ 值最高可达 $98 \%$, 随后用该底 物与二苯基锌反应, 高选择性地得到了 $\mathrm{S}_{\mathrm{N}} 2$ 取代产物且 产物的 $e e$ 值达 $92 \%$ (Eq. 25).

\section{3 有机铝试剂}

Woodward 首先报道了三烷基铝用于铜催化的烯 丙基烷基化反应中，但是实验结果并不理想. 后来 Hoveyda 等 ${ }^{[53]}$ 将手性配体 L18 用于三烷基铝参与的反应 中, 结果所得产物具有很高的立体选择性 $(\gamma: \alpha=20$ : 1)与对映选择性(产物 $e e>98 \%$ ) (Eq. 26). 最近，乙烯基 铝试剂代替烷基铝被用于烯丙基烷基化反应，实验结果 发现所得产物的 $e e$ 值较高 ${ }^{[54]}$. 


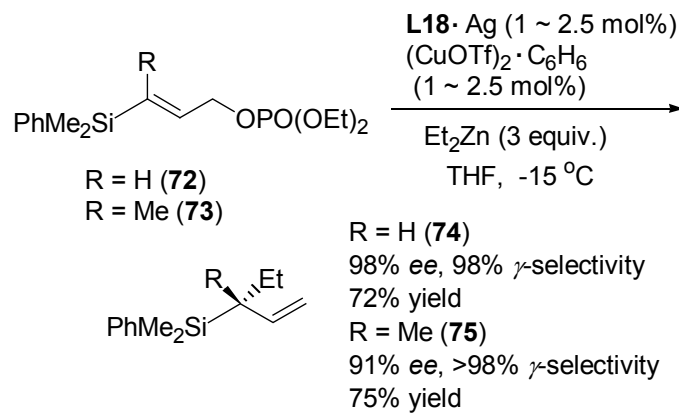

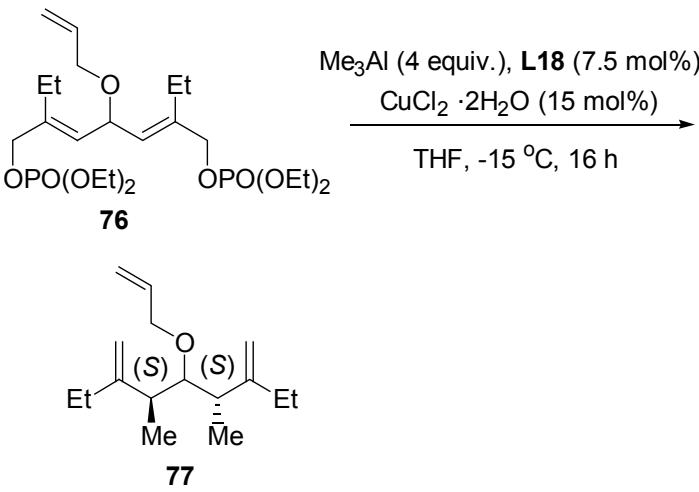

$61 \%$ yield, $>98 \%$ ee

\section{4 结束语}

最近, 铜催化的不对称烯丙基烷基化反应越来越受 到人们的重视, 此类反应具有重要的应用前景. 可用在 不对称烯丙基烷基化反应中的催化剂种类较多, 但是铜 催化剂具有自身的优势(成本较低、催化活性较高), 含 磷配体、多肽基配体、含杂环配体等都可以和铜一起催 化不对称烯丙基烷基化反应，虽然目前报道的手性配体 较多, 但是其应用都存在一定的限度, 没有一种配体具 有通用性, 因此寻找更为有效的手性配体及改进现有的 反应条件以提高反应的速率及不对称合成中产物的对 映选择性, 仍是今后研究的热点课题.

\section{References}

[1] Takeuchi, R. Polyhedron 1999, 19, 557.

[2] Trost, B. M.; Van Vranken, D. L. Chem. Rev. 1996, 96, 395.

[3] Gunasekara, N. G.; Noble, S.; Benfield, P. Drugs 1998, 55, 85.

[4] Miyabe, H.; Takemoto, Y. Synlett 2005, 1641.

[5] Trost, B. M. J. Org. Chem. 2004, 69, 5813.

[6] Wu, Y.-J.; Yang, D.-Q.; Long, Y.-H. Chin. J. Org. Chem. 2009, 29, 1522 (in Chinese). (吴钰娟, 杨定乔, 龙玉华, 有机化学, 2009, 29, 1522.)

[7] Wang, Y.-F.; Zeng, J.-H.; Cui, X.-R. Chin. J. Org. Chem. 2010, 30, 181 (in Chinese). (王晔峰, 曾京辉, 崔晓瑞, 有机化学, 2010, 30, 181.)

[8] Xu, H.-J.; Man, Q.-S.; Lin, Y.-C.; Li, Y.-Y.; Feng, Y.-S. Chin. J. Org. Chem. 2010, 30, 9 (in Chinese). (许华建，蔄秋石，林义成，李源源，冯乙巳，有机化学，2010, 30,9.)
[9] Qin, Y.-C.; Peng, Q. Chin. J. Org. Chem. 2011, 31, 1169 (in Chinese).

(秦元成, 彭强, 有机化学, 2011, 31, 1169.)

[10] Yorimitsu, H.; Oshima, K. Angew. Chem., Int. Ed. 2005, 44, 4435.

[11] Kar, A.; Argade, N. P. Synthesis 2005, 2995.

[12] Goering, H. L.; Singleton Jr, V. D. J. Am. Chem. Soc. 1976, 98, 7854.

[13] Goering, H. L.; Kantar, S. S. J. Org. Chem. 1984, 49, 422.

[14] Goering, H. L.; Singleton Jr, V. D. J. Am. Chem. Soc. 1983, 48, 1531.

[15] Corey, E. J.; Boaz, N. W. Tetrahedron Lett. 1984, 25, 3063.

[16] Bertz, S. H.; Dabbagh, G.; Mujsce, A. M. J. Am. Chem. Soc. 1991, 113,631

[17] Berlan, J.; Battioni, J. P.; Koosha, K. J. Organomet. Chem. 1978, $152,359$.

[18] Lipshutz in, B. H. Organomet. Synth. 1994, 4, 283.

[19] Persson, E. S. M.; van Klaveren, M.; Grove, D. M.; Bäckvall, J. E.; van Koten, G. Chem. Eur. J. 1995, 1, 351 .

[20] Karlström, A. S. E.; Bäckvall, J. E. Chem. Eur. J. 2001, 7, 1981.

[21] van klaveren, M.; Persson, E. S. M.; del Villar, A.; Grove, D. M.; Bäckvall, J.-E.; van Koten, G. Tetrahedron Lett. 1995, 36, 3059.

[22] Meuzwlaar, G. J.; Karlstrom, A. S. E.; Van Klaveren, M.; Persson, E. S. M.; Del Villar, A.; van Koten, G.; Bäckvall, J. E. Tetrahedron 2000, 56, 2895.

[23] Karlström, A. S. E.; Huerta, F. F.; Meuzelaar, G. J. Bäckvall, J. E. Synlett 2001, 923.

[24] Alexakis, A.; Malan, C.; Lea, L.; Benhaim, C.; Fournioux, X. Synlett 2001, 927.

[25] Alexakis, A.; Croset, K. Org. Lett. 2002, 4, 4147.

[26] Tissot-Croset, K.; Polet, D.; Alexakis, A. Angew. Chem., Int. Ed. 2004, 43, 2426.

[27] Tissot-Croset, K.; Polet, D.; Gille, S.; Hawner, C.; Alexakis, A. Synthesis 2004, 2586.

[28] Tissot-Croset, K.; Alexakis, A. Tetrahedron Lett. 2004, 45, 7375.

[29] Falciola, C. A.; Tissot-Croset, K.; Alexakis, A. Angew. Chem., Int. Ed. 2006, 45, 5995.

[30] Tominaga, S.; Oi, Y.; Kato, T.; An, D. K.; Okamato, S. Tetrahedron Lett. 2004, 45, 5585.

[31] Okamato, S.; Tominaga, S.; Saino, N.; Kase, K.; Shimoda, K. J. Organomet. Chem. 2005, 690, 6001.

[32] Lopez, F.; Van Zijl, A. W.; Minnaard, A. J.; Feringa, B. L. Chem. Commun. 2006, 409.

[33] Van Zijl, A. W.; Lopez, F.; Minnaard, A. J.; Feringa, B. L. J. Org. Chem. 2007, 72, 2558.

[34] Geurts, K. Fletcher, S. P.; Feringa, B. L. J. Am. Chem. Soc. 2006. 128,15572 .

[35] Teichert, J. F.; Zhang, S. Y.; van Zijl, A. W.; Slaa, J. W.; Minnaard, A. J.; Feringa, B. L. Org. Lett. 2010, 12, 4658.

[36] Selim, K. B.; Matsumoto, Y.; Yamada, K.; Tomioka, K. Angew. Chem., Int. Ed. 2009, 48, 8733.

[37] Mastral, M. F.; Horst, B.; Minnaard, A. J.; Feringa, B. L. Chem. Commun. 2011, 47, 5843.

[38] Dubner, F.; Knochel, P. Angew. Chem., Int. Ed. 1998, 38, 379.

[39] Dubner, F.; Knochel, P. Tetrahedron Lett. 2000, 41, 9233.

[40] Malda, H.; van Zijl, A. W.; Arnold, L. A.; Feringa, B. L. Org. Lett. 2001, 3, 1169 .

[41] van Zijl, A. W.; Arnold, L. A.; Minnaard, A. J.; Feringa, B. L. Adv. Synth. Catal. 2004, 346, 413.

[42] Borner, C.; Goldsmith, P. J.; Woodward, S.; Gimeno, J.; Gladiali, S.; Ramazzotti, D. Chem. Commun. 2000, 2433.

[43] Bennet, S. M. W.; Brown, S. M.; Cunningham, A.; Dennis, M. R.; Muxworthy, J. P.; Oakley, M. A.; Woodward, S. Tetrahedron 2000, 
$56,2847$.

[44] Goldsmith, P. J.; Teat, S. J.; Woodward, S. Angew. Chem., Int. Ed. 2005, 44, 2235.

[45] Luchaco-Cullis, C. A.; Mizutani, H.; Murphy, K. E.; Hoveyda, A. H. Angew. Chem., Int. Ed. 2001, 40, 1456.

[46] Murphy, K. E.; Hoveyda, A. H.; J. Am. Chem. Soc. 2003. 125, 4690.

[47] Hoveyda, A. H.; Hird, A. W.; Kacprzynski, M. A. Chem. Commun. 2004, 1799.

[48] Kacprzynski, M. A. Hoveyda, A. H. J. Am. Chem. Soc. 2004. 126, 10676.

[49] Piarulli, U.; Daubos, P.; Claverie, C.; Roux, M.; Gennari, C. Angew.
Chem., Int. Ed. 2003, 42, 234.

[50] Piarulli, U.; Claverie, C.; Daubos, P.; Gennari, C.; Minnaard, A. J.; Feringa, B. L. Org. Lett. 2003, 5, 4493.

[51] Larsen, A. O.; Leu, W.; Oberhuber, C. N.; Campbell, J. E.; Hoveyda, A. H. J. Am. Chem. Soc. 2004, 126, 11130.

[52] Kacprzynski, M. A.; May, T. L.; Kazane, S. A.; Hoveyda, A. H. Angew. Chem., Int. Ed. 2007, 46, 4554.

[53] Gillingham, D. G.; Hoveyda, A. H. Angew. Chem., Int. Ed. 2007, 46,3860 .

[54] Lee, Y.; Akiyama, K.; Gillingham, D. G.; Brown, M. K.; Hoveyda, A. H. J. Am. Chem. Soc. 2008. 130, 446.

(Qin, X.) 